\title{
Experimental Study on Effects of Non-spherisity of Smoke Particles on the Light Scattering Characteristics
}

\author{
QIYUAN XIE HEPING ZHANG YONGMING ZHANG and LIFENG QIAO \\ State Key Laboratory of Fire Science, USTC, Hefei, PR China, 230027
}

\begin{abstract}
Light scatting characteristic of smoke particles plays an important role for the developing of photoelectric smoke detection technology. An experimental setup is built to measure the Stokes scattering matrix for typical smoke particles. The results show that the non-spherical properties of smoke particles play an important role on their light scattering characteristic. It is also shown that the measured Stokes scattering matrixes are nearly block-diagonal with symmetric relationships between some elements, which suggest that the smoke particles may be randomly oriented. Through comparing the experimental results and the previous theoretical analysis, it is suggested that spheroid models are more suitable than spherical models to analyze the light scattering by smoke particles. The distinctive features of the light scattering by different kinds of smoke particles can be presented based on spheroid models.
\end{abstract}

KEYWORDS: detection, light scattering characteristic, smoke particles, non-spherical shape model

\section{INTRODUCTION}

The objective of the development of the fire detection technology is to accurately detect the fires as early as possible in order to protect the people and reduce the loss of properties. Smoke particles are one of the most popular and typical fire parameters at the early stages of fires. The technologies of smoke detection, including those methods based on the ionization and photoelectric etc., play important parts in the field of fire detection ${ }^{[1-3]}$. Currently, since ionization smoke detectors use a radioactive source as a part of their working principle, they are being replaced by photoelectric detectors in the market, which are based on light scattering principles ${ }^{[4]}$. The studies of light scattering by smoke particles, as well as some nuisance particles play a key role on the development of photoelectric smoke detectors. In the previous works, the spherical model, namely the Lorenz-Mie theory, was generally used to analyze the light scattering by

smoke particles ${ }^{[5-7]}$. And most of these works only focus on the angular distribution of the intensity of scattered light. However, the SEM micrographs of smoke particles indicate that they are not spherical but branched chain-like and composed of small primary particles ${ }^{[8-12]}$. Based on the Maxwell electromagnetic scattering theory, the morphologies of smoke particles have considerable impacts on the light scattering. In addition, the $4 \times 4$ Stokes scattering matrixes detailedly describe the properties of light scattering by smoke particles. Besides the intensity of scattered light, more information of the light scattering by smoke particles can be found out by analyzing the Stokes scattering matrix. In this case, photoelectric smoke detectors based on new working principles can be designed. Qiyuan Xie et al theoretically analyzed the Stokes Scattering Matrix of smoke particles with spherical and spheroid models based on the SEM micrograph of smoke particles ${ }^{[13]}$. The results show that after averaging of the orientation and size, the nonsphericity of smoke particles has a considerable effect on their light scattering. Additionally, the nonsphericity of gray smoke particles generated from smoldering fires is more important than soot from flaming fires for analyzing the light scattering. Therefore, in this paper, an experimental setup is designed. Based on this setup, the whole Stokes scattering matrixes are measured for three kinds of typical smoke particles, namely smoldering cottons, flaming n-heptane fires and smoldering sandalwoods. The light scattering properties of smoke particles are analyzed and compared with the theoretical results.

\section{THEORY}

The properties of light scattering by small particles, such as smoke aggregates, can be analyzed theoretically through solving the Maxwell equations in the time or frequency domain ${ }^{[14]}$. Using the Stokes vector $\{I, Q, U, V\}^{\mathrm{T}}$ to represent the incident and scattered light, the $4 \times 4$ Stokes scattering matrix $\mathbf{F}(\theta)$ describes the transformation of the incident light into the scattered light. In practical applications, the most popular case is that randomly oriented particles form a macroscopically isotropic and mirror-symmetric medium. Accordingly, the ensemble-averaged characteristics of light scattering by these particles are 
described by a block-diagonal Stokes scattering matrix, as shown in Equation(1) ${ }^{[14]}$.

$$
\left[\begin{array}{l}
I^{s c a}(\theta) \\
Q^{s c a}(\theta) \\
U^{s c a}(\theta) \\
V^{s c a}(\theta)
\end{array}\right]=\frac{1}{r^{2}} \frac{1}{k^{2}} \cdot\left[\begin{array}{llll}
F_{11}(\theta) & F_{12}(\theta) & 0 & 0 \\
F_{12}(\theta) & F_{22}(\theta) & 0 & 0 \\
0 & 0 & F_{33}(\theta) & F_{34}(\theta) \\
0 & 0 & -F_{34}(\theta) & F_{44}(\theta)
\end{array}\right] \cdot\left[\begin{array}{l}
I^{i n c} \\
Q^{i n c} \\
U^{i n c} \\
V^{i n c}
\end{array}\right],
$$

where $0^{\circ} \leq \theta \leq 180^{\circ}$ is the scattering angle. The angle dependence of various elements of the Stokes scattering matrix either provide a direct measure of or are indicative of the physical properties of the scattering medium. For instance, the $(1,1)$ element of the Stokes scattering matrix, $F_{11}(\theta)$, represents the transformation of total intensity of incident light. The $F_{12}(\theta)$ represents the depolarization of linearly polarized light parallel and perpendicular to the scattering plane. The $F_{22}(\theta)$ represents the transformation of linearly polarized scattered light $\left( \pm 90^{\circ}\right)$ to linearly polarized scattered light $\left( \pm 90^{\circ}\right)$. Finally, considering a special case, namely, for a homogeneous spherical particle, there are always $F_{22}(\theta) \equiv F_{11}(\theta)$ and $F_{33}(\theta) \equiv F_{44}(\theta)$, which is the results of the Lorenz-Mie theory. Therefore, the deviation of $F_{22}(\theta)$ from $F_{11}(\theta)$ is an important indication of the presence of non-spherical particles in theoretical analyses and experimental measurements. Similarly, the deviation of $F_{44}(\theta)$ from $F_{33}(\theta)$ is another illustration of the non-spherical particles for light scattering.

\section{EXPERIMENTAL APPARATUS}

An experimental setup was built in the State Key Laboratory of Fire Science (SKLFS) of the University of Science and Technology of China (USTC) to measure the Stokes scattering matrix of smoke particles. The schematic overview and the measuring principle of this experimental setup are shown in Fig. 1. A beam with wavelength of $635 \mathrm{~nm}$ produced by a semiconductor laser passes through a linear polarizer and an electro-optic modulator before it illuminates the smoke particles contained in a jet stream. In this way, the measuring of light scattering by smoke particles can be carried out on-line. The EOM is used to modulate the phase of the incident laser. The modulation frequency used here is $20 \mathrm{MHz}$.Light scattered by the smoke particles at a scattering angle $\theta$ may optionally pass through a quarter-wave plate and a polarization analyzer before its flux is measured by a photomultiplier (PMT1). A rotating plate, which is controlled by a motor through a computer, is used to drive an arm to rotate around the light scattering center of smoke particles. In this way, the angular distribution of scattered light by smoke particles is measured through the PMT1. This is the most distinctive feature between the experimental setup developed in the SKLFS and the one in the Free University of the Netherlands ${ }^{[15-18]}$. By adjusting the three-dimensional position of the nozzle of the smoke particles and the orientation of the collimating pinhole in front of the PMT1, the pinhole can collimate the scattering center of smoke particles at every scattering angle $\theta$. The diameter of the two pin-holes is $1 \mathrm{~mm}$. The distance between the two pin-holes is $50 \mathrm{~mm}$. The distance from the first or nearer pin-hole to the center-line is $500 \mathrm{~mm}$. Another photomultiplier (PMT2) is used to monitor the flux of the scattered light at a fixed scattering angle on the other side of the rotating plate. The DC signals measured by the PMT2 are used to correct for the fluctuation of smoke particles in the jet stream. The signals measured by PMT1 and PMT2 are recorded and stored in the computer through the Lock-in amplifier. The modulation and lock-in detection is done so that the weak signal due to the light scattering from the smoke particles can be measured.

Using Stokes parameters, the Stokes vector of the scattered light reaching the PMT1 can be written as

$$
\left[\begin{array}{l}
I^{s c a}(\theta) \\
Q^{s c a}(\theta) \\
U^{s c a}(\theta) \\
V^{s c a}(\theta)
\end{array}\right]=c_{1} \cdot \mathbf{A}\left(\gamma_{A}\right) \cdot \mathbf{Q}\left(\gamma_{Q}\right) \cdot \mathbf{F}(\theta) \cdot \mathbf{M}\left(\gamma_{M}\right) \cdot \mathbf{P}\left(\gamma_{P}\right) \cdot\left[\begin{array}{l}
I^{i n c} \\
Q^{i n c} \\
U^{i n c} \\
V^{i n c}
\end{array}\right],
$$


where $\mathbf{I}^{\text {inc }}$ is the Stokes vector of the beam leaving the light source; $c_{1}$ is a real constant; and $\mathbf{A}, \mathbf{Q}, \mathbf{M}$ and $\mathbf{P}$ are $4 \times 4$ Muller matrices of the analyzer, quarter-wave plate, modulator and polarizer, respectively. The orientation angles $\gamma_{A}, \gamma_{Q}, \gamma_{M}$ and $\gamma_{P}$ of the corresponding optical components are the angles between their optical axes and the reference plane. Here it is assumed that the scattering plane acts as the reference plane for these orientation angles and the Stokes parameters. This plane coincides with the horizontal plane in the experimental setup. The $\mathbf{F}(\theta)$ is the to-be-measured $4 \times 4$ Stokes scattering matrix of the smoke particles.

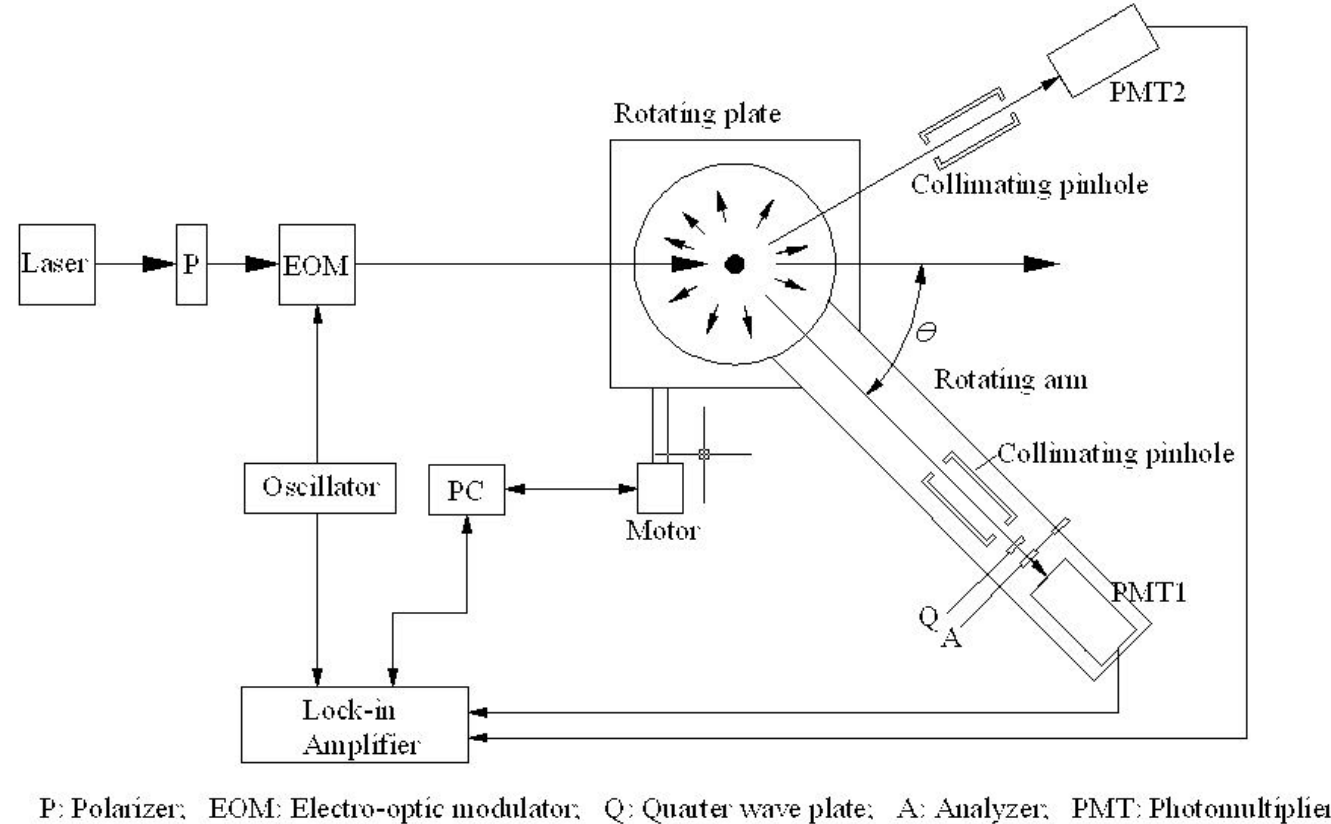

Fig. 1. Schematic overview of the experimental setup built in the SKLFS

By adjusting the amplitude of the modulation voltage of the EOM and analyzing the four Muller matrices in Equation (2), the flux reaching the PMT1 can be written as

$I^{s c a}(\theta)=c_{2}\left[D C(\theta)+2 J_{1}\left(\phi_{0}\right) S(\theta) \sin \omega t+2 J_{2}\left(\phi_{0}\right) C(\theta) \cos 2 \omega t\right]$,

where $c_{2}$ is a constant for a specific optical arrangement, $2 J_{1}\left(\phi_{0}\right)=1.03830,2 J_{2}\left(\phi_{0}\right)=0.86350$ and higher order terms have been omitted. The coefficients $D C(\theta), S(\theta)$ and $C(\theta)$ respectively correspond to the $d c$, $\sin \omega t$ and $\cos 2 \omega t$ components of the measured signal of PMT1.

Table 1 shows eight combinations of the orientation angles of the four optical elements in this setup. The corresponding relationship between the elements of the scattering matrix and the measured signal components is also indicated. Through using lock-in amplifier the $\sin \omega t$ and the $\cos 2 \omega t$ components can be separated from the total detected signal of PMT1. These two components plus the constant $(d c)$ part of the detected signal of PMT1 are sufficient to determine the angular distributions of all elements of the scattering matrix for smoke particles. Here not only the six independent elements shown in Equation(1), but also the other ten elements are measured experimentally. In this case, the measured 16 curves are analyzed to consider whether the real smoke particles are randomly oriented or not. For more detailed information on the measuring principle of this experimental setup, please refer to the descriptions of setups in SKLFS and in Amsterdam ${ }^{[15-18]}$. 
Table 1. Relationship between elements of scattering matrix and the measured signal components for eight combinations of the orientation angles of the four optical elements.

\begin{tabular}{cccccccc}
\hline Comb. & $\gamma_{P}$ & $\gamma_{M}$ & $\gamma_{Q}$ & $\gamma_{A}$ & $D C(\theta)$ & $S(\theta)$ & $C(\theta)$ \\
\hline 1 & $0^{\circ}$ & $-45^{\circ}$ & -- & -- & $F_{11}$ & $-F_{14}$ & $F_{12}$ \\
2 & $0^{\circ}$ & $-45^{\circ}$ & -- & $0^{\circ}$ & $F_{11}+F_{21}$ & $-\left(F_{14}+F_{24}\right)$ & $F_{12}+F_{22}$ \\
3 & $0^{\circ}$ & $-45^{\circ}$ & -- & $45^{\circ}$ & $F_{11}+F_{31}$ & $-\left(F_{14}+F_{34}\right)$ & $F_{12}+F_{32}$ \\
4 & $0^{\circ}$ & $-45^{\circ}$ & $0^{\circ}$ & $45^{\circ}$ & $F_{11}+F_{41}$ & $-\left(F_{14}+F_{44}\right)$ & $F_{12}+F_{42}$ \\
5 & $45^{\circ}$ & $0^{\circ}$ & -- & -- & $F_{11}$ & $-F_{14}$ & $F_{13}$ \\
6 & $45^{\circ}$ & $0^{\circ}$ & -- & $0^{\circ}$ & $F_{11}+F_{21}$ & $-\left(F_{14}+F_{24}\right)$ & $F_{13}+F_{23}$ \\
7 & $45^{\circ}$ & $0^{\circ}$ & -- & $45^{\circ}$ & $F_{11}+F_{31}$ & $-\left(F_{14}+F_{34}\right)$ & $F_{13}+F_{33}$ \\
8 & $45^{\circ}$ & $0^{\circ}$ & $0^{\circ}$ & $45^{\circ}$ & $F_{11}+F_{41}$ & $-\left(F_{14}+F_{44}\right)$ & $F_{13}+F_{43}$ \\
\hline
\end{tabular}

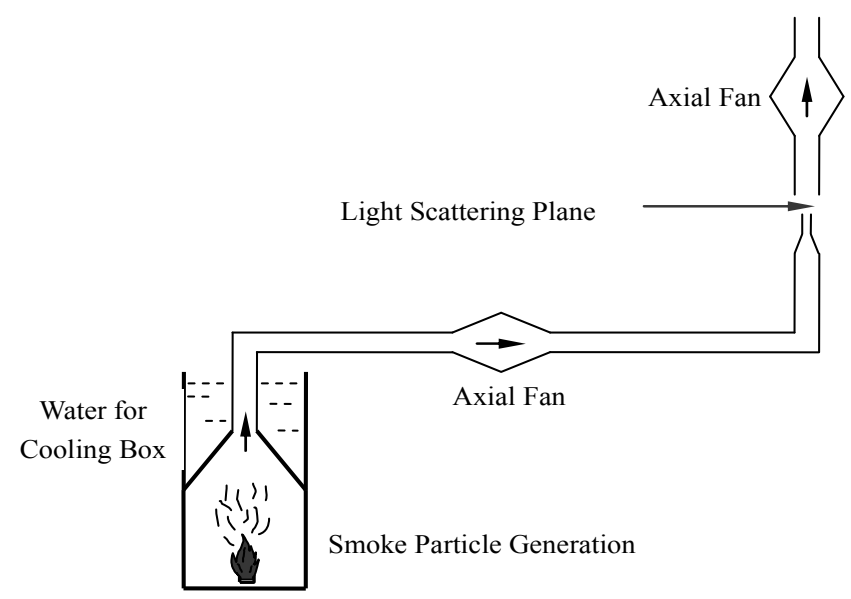

Fig. 2. Smoke particles generation box and transportation tubes

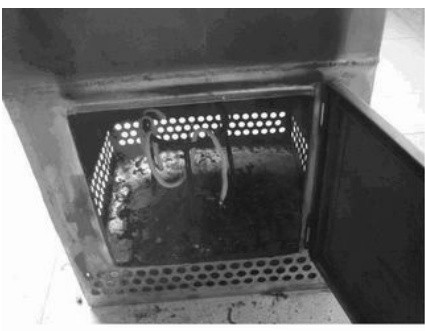

(a)Smoldering cottons

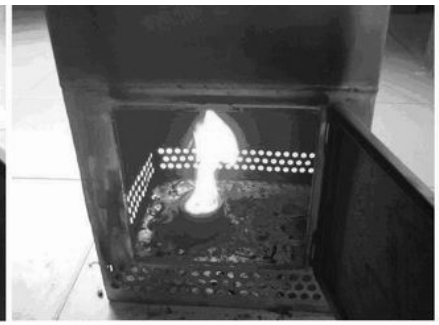

(b) Flaming heptane

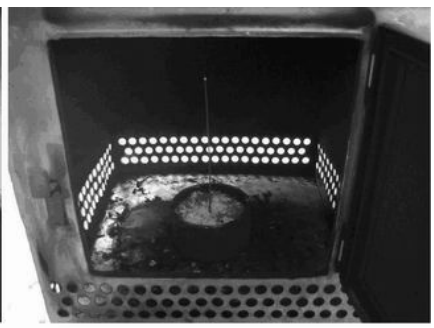

(c) Smoldering sandalwoods

Fig. 3. Smoke generation of smoldering cottons, flaming heptanes, smoldering sandalwoods

For each experiment, a proper smoke generation box also play an important role in the measurements of Stokes scattering matrix for smoke particles. It takes quite a few minutes to measure the angular distribution of the scattered light for each combination. A smoke jet, which is as stable as possible, is necessary for the measurement although the PMT2 is used to monitor the scattered light at a certain angle for the compensation of the fluctuation of smoke particles. Therefore, as shown in Fig. 2, a smoke generation box and the supplemental transportation tubes are designed. A small water tank is used to cool and protect the box. The generated smoke particles are transported through several axial fans. Figure 2 illustrates that a short cut of smoke jet is exposed for light scattering. The angular distribution of Stokes scattering matrix is measured at the corresponding light scattering plane. The smoke particles are exhausted out immediately after light scattering. The generation for three kinds of typical smoke particles is shown in Fig. 3, respectively. 
As addressed in the EN54 codes ${ }^{[19]}$, smoke particles generated from smoldering cotton fire (TF3) and flaming n-heptane fire (TF5) are representative for two kinds of combustion styles (flaming and smoldering). Additionally, smoke particles from smoldering sandalwoods are typical nonstandard smoke particles in the field of fire detection. The black smoke particles generated from flaming n-heptane fire have relatively larger image part of a refractive index than the gray smoke particles from smoldering cottons and sandalwoods. Figure 4 give the measured angular distributions of 8 elements of Stokes scattering matrix for these three kinds of smoke particles through the above experimental setup. The scattered light is measured for scattering angles between $5^{\circ}$ and $160^{\circ}$ with the step of $0.9^{\circ}$ to get angular distributions of Stokes scattering matrix. Similar to previous theoretical analyses, the ratios $F_{i j}(\theta) / F_{11}(\theta)$ are considered, except for the $F_{11}(\theta)$.

Figure 4(a) gives the normalized angular distributions of $F_{11}(\theta)$ for smoke particles generated from smoldering cottons, flaming n-heptane and smoldering sandalwoods. As can be seen in Figure 4(a), the measured $F_{11}(\theta)$ curve for the flaming n-heptane is different from those for the smoldering cottons and smoldering sandalwoods. At the angles smaller than $60^{\circ}$, the normalized intensity of scattered light for the two smoldering fires is larger than those for flaming n-heptane. However, at the scattering angles larger than $80^{\circ}$, there is only a little difference among the three curves. It is suggested that the photoelectric smoke detectors based on forward-scattering principles respond more sensitively to gray smoke particles than to black smoke particles. The detectors based on backward-scattering principles show similar sensitivity to gray and black smoke particles.

As can be seen in Fig. 4(b), all the measured $F_{22}(\theta) / F_{11}(\theta)$ curves for the three kinds of smoke particles obviously deviate from " 1 ". This is a key indication for the obvious effects of the non-spherical property of the smoke particles on their light scattering. Through comparing the measured $F_{22}(\theta) / F_{11}(\theta)$ curves for the three kinds of smoke particles and the theoretically calculated curves based on spherical and spheroid models, it suggests that the spheroid models are more suitable to analyze the light scattering by smoke particles than spherical models. Furthermore, the $F_{22}(\theta) / F_{11}(\theta)$ curves for the two kinds of gray smokes particles show more deviation from "1" than that for black smoke particles, especially at the backward scattering angles. The minimum values of the $F_{22}(\theta) / F_{11}(\theta)$ curves for the two kinds of gray smoke particles are about 0.25 , while the minimum value for the black smoke particles is about 0.5 . These experimental results suggest that the non-spherical property of the gray smoke particles generated from smoldering fires plays more considerable roles on the light scattering than the black smoke particles generated from flaming fires. In addition, it is also indicated from the previous theoretically calculated results based on spheroid models that the non-spherical property of the smoke particles with smaller image part of refractive index show more effect on their light scattering ${ }^{[13]}$. In this case, it is suggested that the experimental results agree well with the theoretically calculated results based on the spheroid models.

Figure 4 (c) and (d) respectively give the measured $-F_{12}(\theta) / F_{11}(\theta)$ and $-F_{21}(\theta) / F_{11}(\theta)$ curves for the three kinds of smoke particles. As can be seen in these figures, the measured $-F_{12}(\theta) / F_{11}(\theta)$ and $-F_{21}(\theta) / F_{11}(\theta)$ curves are quite similar to each other for every kind of smoke particles. There is an obvious peak values in each curve. Furthermore, the peak value in the curve for the black smoke particles, namely about 0.6 is larger than the corresponding peak values for two kinds of gray smoke particles, namely about 0.25 . These characteristics of the measured $-F_{12}(\theta) / F_{11}(\theta)$ and $-F_{21}(\theta) / F_{11}(\theta)$ curves also fit well with the previous calculated results based on spheroid models for smoke particles with different refractive indexes ${ }^{[13]}$. It is suggested again that spheroid models may be more suitable to calculate the light scattering by smoke particles.

Figure 4 (e) and (f) show the measured $F_{33}(\theta) / F_{11}(\theta)$ and $F_{44}(\theta) / F_{11}(\theta)$ curves. At the scattering angles near to $5^{\circ}$, both the $F_{33}(\theta) / F_{11}(\theta)$ and $F_{44}(\theta) / F_{11}(\theta)$ values are about 0.9 . As the increasing of scattering angles, both $F_{33}(\theta) / F_{11}(\theta)$ and $F_{44}(\theta) / F_{11}(\theta)$ values decrease and reach minimum values at scattering angles larger than $90^{\circ}$. In addition, at those large scattering angles, the $F_{44}(\theta) / F_{11}(\theta)$ values are greater than the $F_{33}(\theta) / F_{11}(\theta)$ values. Here the influence of non-spherical property of the smoke particles on their light scattering is illustrated again. 


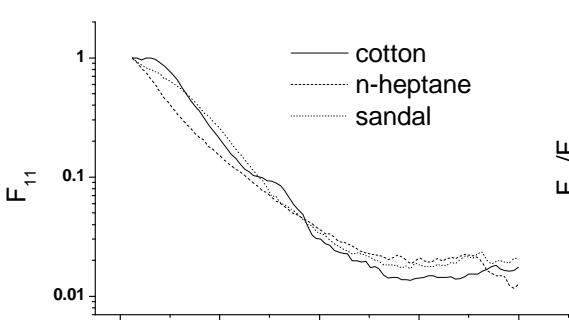

(a)

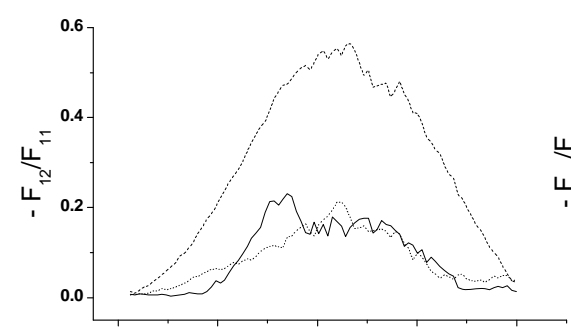

(c)

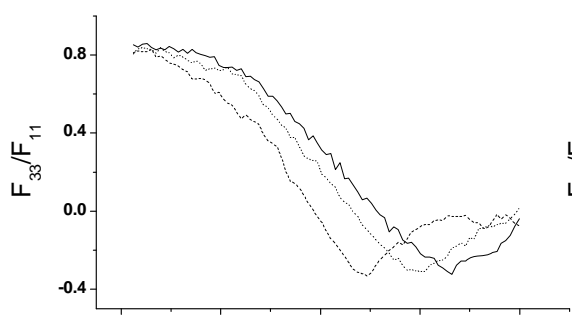

(e)

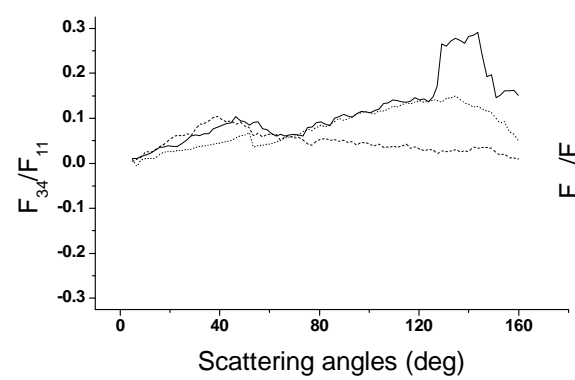

(g)

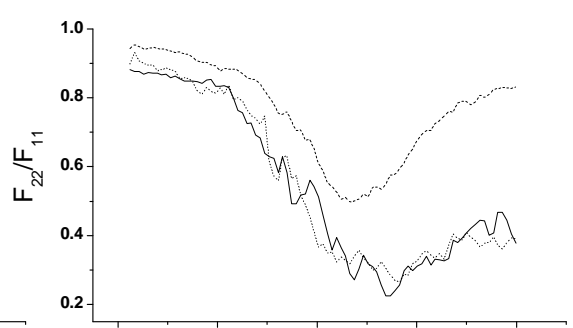

(b)

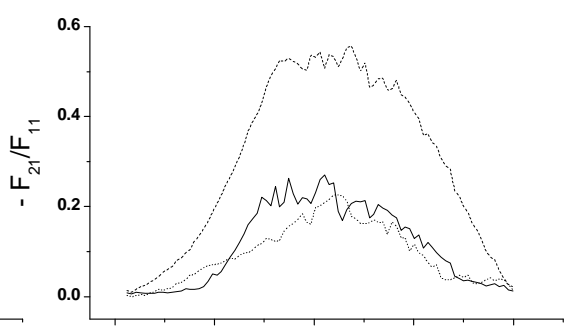

(d)

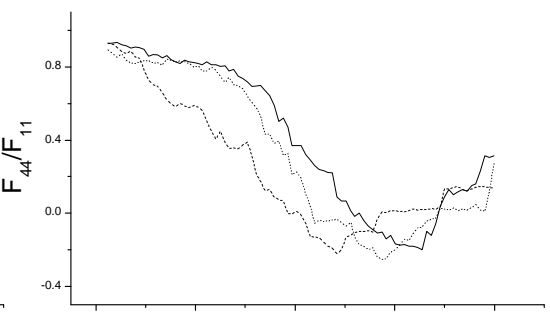

(f)

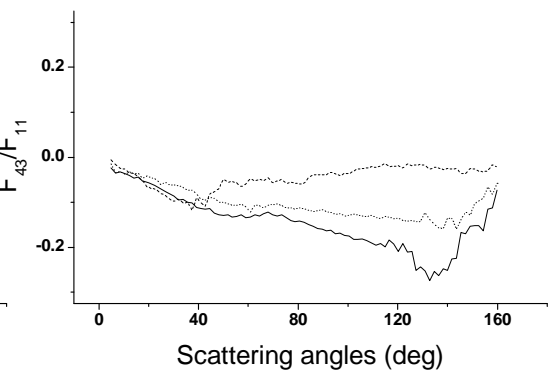

(h)

Fig. 4. Measured stokes scattering matrix element $F_{11}(\theta)$, and element ratios $F_{22}(\theta) / F_{11}(\theta)$ et al. for smoke particles generated from smoldering cottons, flaming n-haptane and smoldering sandalwoods.

Figure $4(\mathrm{~g})$ and (h) respectively give the measured $F_{34}(\theta) / F_{11}(\theta)$ and $F_{43}(\theta) / F_{11}(\theta)$ curves for the three kinds of smoke particles. As can be seen from these figures, the $F_{34}(\theta) / F_{11}(\theta)$ curve is symmetrical to the $F_{43}(\theta) / F_{11}(\theta)$ curve for each kind of smoke particles. Figure $2(\mathrm{~g})$ indicates that there are peak values at the backward scattering angles in the $F_{34}(\theta) / F_{11}(\theta)$ curves for the two kinds of smoke particles generated from smoldering fires. However, a corresponding peak value is at the forward scattering angles in the $F_{34}(\theta) / F_{11}(\theta)$ curves for the flaming n-heptane fire. 
The measured angular distributions of the other eight element ratios of Stokes scattering matrix for these three kinds of smoke particles are rather small and almost equal to zero. The highest absolute value of the measured element ratio is less than 0.02. In addition, as indicated above, the measured $-F_{12}(\theta) / F_{11}(\theta)$ and $-F_{21}(\theta) / F_{11}(\theta)$ curves are similar to each other, as well as $F_{34}(\theta) / F_{11}(\theta)$ and $F_{43}(\theta) / F_{11}(\theta)$ curves are symmetrical to each other for every kind of smoke particles. These suggest that the measured Stokes scattering matrixes of the three kinds of smoke particles are block-diagonal with some symmetric relationships, as described in Equation (1). In this case, the real smoke particles may be regarded as randomly oriented.

As mentioned above, Qiyuan Xie et al. analyzed the Stokes Scattering Matrix of smoke particles with different refractive index and size based on spherical and spheroid models. Comparing the above experimental results with those theoretical analyses based on spheroid models ${ }^{[13]}$, it illustrates that the nonspherical property of real smoke particles plays a considerable role on their light scattering, especially for the gray smoke particles generated from smoldering fires. In addition, the experimental measurements fit relatively well with the theoretical results based on spheroid shape models. The distinctive features between light scattering by gray and black smoke particles can be analyzed based on spheroid models. Finally, the real smoke particles may be considered to be randomly oriented.

\section{CONCLUSIONS}

In this paper, an experimental setup is built to measure the angular distributions of the $4 \times 4$ Stokes scattering matrix for real smoke particles. The Stokes scattering matrixes of the smoke particles generated from smoldering cottons, flaming n-heptane and smoldering sandalwoods are measured. The experimental measurements show that the $F_{22}(\theta) / F_{11}(\theta)$ curves for three kinds of smoke particles obviously deviate from " 1 ", especially for those generated from smoldering fires. It is illustrated that the non-spherical properties of smoke particles play an important role on their light scattering. In addition, the measured $4 \times 4$ Stokes scattering matrixes are approximately block-diagonal with symmetric relationships between some elements, which suggest that the smoke particles are randomly oriented. Through comparing the experimental and the previous theoretical results, it is suggested that spheroid models are more suitable than spherical models to analyze the light scattering by smoke particles. The distinctive features of the light scattering by different kinds of smoke particles can also be analyzed based on spheroid models.

Future work should focus on the experimental measurements of the Stokes scattering matrixes for more kinds of smoke particles and the nuisance particles for smoke detections, such as the dusts, cooking fumes, steam etc. The design of new photoelectric smoke detectors based on the experimental measured Stokes scattering matrixes is also very important for the fire protection engineering.

\section{REFERENCES}

[1] Luck H. Remarks on the state of the art in automatic fire detection, Fire Safety Journal, 1997, 29(23): 77-85, doi:10.1016/S0379-7112(96)00030-6

[2] Rexfort C. Combination of a fire model and a smoke sensor model, Fire Safety Journal, 2006, 41(4): 252-257, doi:10.1016/j.firesaf.2005.12.006

[3] Siebel R. Test of fire detection algorithms using artificially generated events, Fire Safety Journal, 2006, 41(4): 258-265, doi:10.1016/j.firesaf.2006.01.004

[4] Fujisawa T, Suzuki T, Yoshikawa Y and Ohkuma S. Optical Smoke Detector using dual light spectrum, 13th International Conference on Automatic Fire Detection, AUBE'04, 2004: 527-536.

[5] Mie G. Contributions on the Optics of Turbid Media, Particularly Colloidal Metal Solutions, Annalen der Physik, 1908, Series , No. 3, 377-445.

[6] Zhao JH, Yuan HY, Fan WC, Chen T. Characterization of the classification characteristic for fire smoke by the extinction coefficient ratio, ACTA PHYSICA SINICA,2002, 51 (3): 700-704.

[7] Shu XM, Study of Fire Smoke Particle Diameter Measurements and Light Scattering Characteristics, $\mathrm{PhD}$ thesis, University of Science and Technology of China, China, 2004. 
[8] Xie QY, Zhang YM and Yuan HY. Preliminary Comparison on the Morphologies of Smoke Particles and Dusts through SEM Imaging, Journal of Applied Fire Science, 2003-2004, 12(3):195202.

[9] Koylu UO, Faeth GM and Farias TL et al. Fractal and Projected Structure Properties of Soot Aggregates, Combustion and Flame, 1995, 100: 621-633, doi:10.1016/0010-2180(94)00147-K

[10] Dobbins RA and Megaridis CM. Morphology of Flame-Generated Soot As Determined by Thermophoretic Sampling, Langmuir, 1987,3:254-259, doi:10.1021/la00074a019

[11] Samson RJ, Mulholland GW and Gentry JW. Structural Analysis of Soot Agglomerates, Langmuir, 1987,3(2):272-281, doi:10.1021/la00074a022

[12] Sorensen CM and Feke GD. The Morphology of Macroscopic Soot, Aerosol Science and Technology, 1996,25:328-337, doi:10.1080/02786829608965399

[13] Xie QY, Zhang HP, Wan YT et al. Characteristics of light scattering by smoke particles based on spheroid models, Journal of Quantitative Spectroscopy and Radiative Transfer, Vol.107 (1), 2007, 72-82, doi:10.1016/j.jqsrt.2007.01.059

[14] Mishchenko M I, Travis L D and Lacis A A. Scattering, Absorption, and Emission of Light by Small Particle, Cambridge University Press, 2002.

[15] Volten H, De Haan JF, Hovenier JW et al. Laboratory measurements of angular distributions of light scattered by phytoplankton and silt. Limnol, Oceanogr 1998: 43: 1180-97.

[16] Stammes P. Light scattering properties of aerosols and the radiation inside a planetary atmosphere, Ph.D Thesis, Free University, Amsterdam, 1989.

[17] Kuik F. Single scattering of light by ensembles of particles with various shapes. Ph.D Thesis, Free University, Amsterdam, 1992.

[18] Xie QY. Study on the Model of Light Scattering by Smoke Particles, PhD thesis, University of Science and Technology of China, 2006.

[19] EN54: Components of Automatic Fire Detection Systems, part 9, fire sensitivity test, 1998. 